\title{
Titel/Title: Gesellschaftliche Integrationsprobleme im Spiegel soziologischer Gegenwartsdiagnosen
}

Autor*innen/Author(s): Uwe Schimank

Veröffentlichungsversion/Published version: Postprint

Zeitschriftenartikel/Journal article

\section{Empfohlene Zitierung/Recommended citation:}

Schimank, Uwe (2000): Gesellschaftliche Integrationsprobleme im Spiegel soziologischer Gegenwartsdiagnosen. In: Berliner Journal für Soziologie, 10, 449-469.

Verfügbar unter/Available at:

(wenn vorhanden, bitte den DOI angeben/please provide the DOI if available)

This is a post-peer-review, pre-copyedit version of an article published in Berliner Journal für Soziologie. The final authenticated version is available online at: https://doi.org/10.1007/BF03204366

Zusätzliche Informationen/Additional information:

Der Autor kann kontaktiert werden unter: uwe.schimank@uni-bremen.de 
Uwe Schimank

\section{Gesellschaftliche Integrationsprobleme im Spiegel soziologischer Gegenwarts- diagnosen}

Von Beginn an hat die Soziologie immer auch zeitdiagnostisch gearbeitet. Sie entstand im 19. Jahrhundert, als sich die charakteristischen Strukturen der Moderne unübersehbar in allen gesellschaftlichen Bereichen herausbildeten. Die damals drängende Frage, wie es mit der modernen Gesellschaft weitergehen würde, beschäftigt uns heute, zu Beginn des 21. Jahrhunderts, nicht minder. Im Vordergrund stehen dabei, heute wie damals, Befürchtungen. Ökologische Katastrophen, mit immer zerstörerischeren Waffen geführte Kriege, um sich greifende soziale Entwurzelung und ein immer rücksichtsloserer Egoismus: Das sind einige Stichworte für den sorgenvollen Blick auf die Zukunft der heutigen Gesellschaft. Daneben artikulieren soziologische Zeitdiagnosen auch Zukunftshoffnungen - wenn auch deutlich seltener als Befürchtungen. So wird heute neben den Risiken der Individualisierung auch auf die Chancen verwiesen, die dieser Vorgang den einzelnen Gesellschaftsmitgliedern eröffnet; und ebenso wird der mit vielen Ängsten begleiteten "Globalisierung" dennoch auch manches Positive abzugewinnen versucht.

Soziologische Zeitdiagnosen sind Lesarten des Heute und Morgen. Trotz aller empirischen und theoretischen Unzulänglichkeiten ${ }^{1}$ wird so ein wichtiger Beitrag zur "soziologischen Aufklärung" der modernen Gesellschaft über sich selbst geleistet. Tatsache ist ja, dass ganz verschiedene Arten von Beobachtern der gesellschaftlichen Zustände Zeitdiagnosen erstellen. Literaten, Philosophen, Politiker, Priester, Journalisten und Sozialwissenschaftler - darunter dann auch Soziologen - versuchen sich daran. Nicht nur, dass die Soziologie hier kein Deutungsmonopol besitzt: Sie ist die meiste Zeit nicht einmal die lauteste Stimme im Chor derer, die den Puls der Zeit zu fühlen versuchen.

Mehr noch: Die Soziologie spricht in zeitdiagnostischen Fragen keineswegs mit einer Stimme. An dieser Thematik erweist sich vielmehr die auch ansonsten immer schon teils negativ, teils auch positiv herausgestellte Multiperspektivität des Faches. Damit

1 Die unter anderem Walter Reese-Schäfer (1996) und Hans-Peter Müller (1997) resümieren. 
ist der Anlass des vorliegenden Beitrags angesprochen. Gleichgültig, ob man die Multiperspektivität der Zeitdiagnosen dadurch geprägt sieht, dass verschiedene Diagnosen miteinander unvereinbare Aussagen treffen, oder ob der vorherrschende Eindruck der ist, dass die Diagnosen aneinander vorbei reden: Beide Einschätzungen legen vergleichende Sekundäranalysen der Zeitdiagnosen nahe. Denn bei miteinander unvereinbaren Aussagen muss geklärt werden, worin genau die Unvereinbarkeit besteht und welche der Aussagen zutrifft und welche nicht; oder es müssen unterschiedliche Geltungsbereiche für die Aussagen spezifiziert werden. Und dort, wo Aussagen unverbunden nebeneinander stehen, wäre zu prüfen, ob es nicht doch Zusammenhänge zwischen ihnen gibt, so dass man zu einem geschlosseneren und umfassenderen Bild der Gesellschaft gelangt. Bereits eine im Bereich theoretischer $(\leftarrow \mathbf{p . 1})$ Reflexionen verbleibende, noch gar nicht zur empirischen Prüfung übergehende Sekundäranalyse vermag so zu Erkenntnisfortschritten zu führen.

Im Weiteren soll dies an einer Reihe von soziologischen Gegenwartsdiagnosen, die in den vergangenen zwanzig Jahren in Deutschland, Frankreich, Großbritannien und den Vereinigten Staaten publiziert worden sind (siehe Abbildung 1), angestoßen werden. ${ }^{2}$ In einem ersten Schritt wird die abstrakte theoretische Leitfrage entfaltet, anhand derer die Gegenwartsdiagnosen hier miteinander verglichen werden. Dies ist die Frage nach der Integration der modernen Gesellschaft. Der daran anschließende zweite Schritt stellt dann Thesen zusammen und nebeneinander, die in den verschiedenen Gegenwartsdiagnosen zu den im ersten Schritt entwickelten Dimensionen gesellschaftlicher Integration zu finden sind. Der begrenzte Rahmen dieses Beitrages ermöglicht keine ins Detail gehende theoretische Konfrontation oder Zusammenführung der dargestellten Thesen. Hier geht es lediglich darum, an bestimmten Punkten theoretische Übereinstimmungen, an anderen Punkten theoretische Widersprüche sowie insgesamt die Vielschichtigkeit des sich aus der Zusammenschau der einzelnen Diagnosen ergebenden Bildes aufzuzeigen.

\section{Abbildung 1:}

Deutschland:

Ulrich Beck:

Die Risikogesellschaft (1986)

2 Dieser Beitrag steht im Rahmen eines entsprechenden Vergleichsprojekts, dessen erster Schritt in einer Aufarbeitung der zentralen Argumentationsfiguren jeder einzelnen der betrachteten Gegenwartsdiagnosen war (vgl. Schimank/Volkmann 2000). Nicht alle der aufgelisteten Gegenwartsdiagnosen können im weiteren mit gleicher Aufmerksamkeit angesprochen werden. 
Richard Münch:

Gerhard Schulze:

Jürgen Habermas:

Peter Gross:

Niklas Luhmann:

Wilhelm Heitmeyer (Hrsg.):
Dialektik der Kommunikationsgesellschaft (1991) sowie: Dynamik der Kommunikationsgesellschaft (1995)

Die Erlebnisgesellschaft (1992)

Faktizität und Geltung (1992)

Die Multioptionsgesellschaft (1994)

Die Gesellschaft der Gesellschaft (1997)

Was treibt die Gesellschaft auseinander? (1997a) sowie: Was hält die Gesellschaft zusammen? (1997b)

\section{Frankreich:}

Bruno Latour:

Alain Touraine:

Pierre Bourdieu et al.:
Nous n'avons jamais été modernes (Wir sind nie modern gewesen) (1991)

Critique de la modernité (Critique of modernity) (1992)

La misère du monde (Das Elend der Welt) (1993
Großbritannien:

Anthony Giddens:

Zygmunt Bauman:

Ralf Dahrendorf:
Consequences of Modernity (Konsequenzen der Moderne) (1990)

Modernity and Ambivalence (Moderne und Ambivalenz) (1991)

The Modern Social Conflict (Der moderne soziale Konflikt) (1992)

\footnotetext{
Vereinigte Staaten:

James Coleman:

George Ritzer:

Amitai Etzioni:

Samuel Huntington:

The Asymmetric Society (Die asymmetrische Gesellschaft) (1982)

The McDonaldisation of Society (Die McDonaldisierung der Gesellschaft) (1993) (<-p. 2)

The Spirit of Community (Die Entdeckung des Gemeinwesens) (1993)

sowie: The New Golden Rule (Die Verantwortungsgesellschaft) (1996)

The Clash of Civilizations and the Remaking of World Orders (Der

Kampf der Kulturen. Die Neugestaltung der Weltpolitik im 21. Jahrhun-

dert) (1996)

Richard Sennett:

The Corrosion of Character (Der flexible Mensch) (1998)
}

Man muss nicht soweit gehen, die Frage nach der gesellschaftlichen Integration zur Schlüsselfrage soziologischer Gesellschaftstheorie schlechthin zu erheben. Unbestreitbar ist es eine zentrale gesellschaftstheoretische Frage. Es verwundert deshalb auch nicht, dass letztlich alle hier betrachteten soziologischen Gegenwartsdiagnosen sich mit der Integration der modernen Gesellschaft befassen; nicht wenige stellen dieses Thema explizit in den Mittelpunkt. Am plakativsten ist es in den Untertiteln der von Wilhelm Heitmeyer (1997a; 1997b) zusammengestellten Studien formuliert: "Was treibt die Gesellschaft auseinander?" und "Was hält die Gesellschaft zusammen?"

Doch obwohl sich die soziologische Gesellschaftstheorie im allgemeinen ebenso intensiv um das Integrationsproblem gekümmert hat, wie das die soziologischen 
Zeitdiagnosen der letzten zwanzig Jahre getan haben, fehlt der Soziologie hierfür nach wie vor ein klares Konzept. Ganz abstrakt meint die Redeweise, daß zwei Einheiten miteinander integriert seien, zunächst stets eine wechselseitige „Reduktion der Freiheitsgrade" (Luhmann 1997: 603). Es ist in beiden Einheiten nicht mehr alles möglich, was möglich wäre, existierten sie isoliert voneinander. Entscheidend dafür, von Integration zu sprechen, ist allerdings, daß es sich um solche wechselseitigen Möglichkeitsbeschränkungen handelt, die beiderseitig als funktional für die Selbstreproduktion der Einheiten einzustufen sind. ${ }^{3}$ Bei gesellschaftlicher Integration ist diejenige Einheit, die man als analytische Referenz wählt, die Gesellschaft. Sie steht zu - noch zu klärenden - anderen Einheiten in einem solchen Verhältnis wechselseitiger Limitationen, das die Fähigkeit der Gesellschaft zur dauerhaften - inzwischen sagt man oft: „nachhaltigen" - Selbstreproduktion erhält oder verbessert. ${ }^{4}$ Aber was heißt das genauer?

Zunächst einmal wird schnell offenbar, dass eine Positivumschreibung gesellschaftlicher Integration analytisch nicht weiter führt. Denn wenn ungestörte Integration vor $(\leftarrow$ p. 3) liegt, ist sie zumeist völlig unscheinbar. Sie wird weder von den Gesellschaftsmitgliedern noch von soziologischen Gesellschaftsbeobachtern als solche bemerkt, sondern für selbstverständlich, gewissermaßen als natürliche Ordnung der Dinge, genommen und hingestellt. Und selbst wenn ein hinreichend distanziert reflektierender Beobachter den gegebenen Zustand ungestörter Integration kontrafaktisch als unwahrscheinlich und daher erklärungsbedürftig ansieht, ist damit noch lange nicht gesagt, dass er sich die reibungslose Erfüllung der gesellschaftlichen Reproduktionserfordernisse auch tatsächlich hinsichtlich der zugrunde liegenden Strukturdynamiken korrekt vorzustellen und zu erklären vermag. Störungsfreie Integration ist gewissermaßen eine black box, und nur im Störungsfall vermag man etwas Licht ins Dunkel zu bringen.

\footnotetext{
Luhmann (1997: 601-604) macht genau diese Begriffsverengung dezidiert nicht mit, weil er den Integrationsbegriff sozusagen nicht positiv besetzen will. Gegenüber den von ihm kritisierten normativen Integrationsvorstellungen ist das überzeugend, nicht aber gegenüber einem funktionalistischen Verständnis.

4 Die klassische strukturfunktionalistische Gesellschaftstheorie sprach diesbezüglich von gesellschaftlicher Bestandserhaltung. Dies wurde in der Folgezeit vielfach als heillos verschwommen und ideologisch kritisiert. Insbesondere bleibt bei Gesellschaften als „selbstsubstitutiven Ordnungen“ (Luhmann 1981) unklar, was das soziale Pendant zum biologischen Tod eines Organismus ist. Diese berechtigten Einwände verlieren jedoch an Gewicht, wenn man das Integrationskonzept, wie im folgenden verdeutlicht werden wird, dimensioniert, gradualisiert und negativ bestimmt. Selbstreproduktion heißt des weiteren auch nicht unbedingt Stabilität aller gesellschaftlichen Strukturen, sondern schließt geordneten, gestalteten Wandel mit ein. Im übrigen wird hier die Selbstreproduktionsfähigkeit des jeweiligen Gegenübers der Gesellschaft nicht eigens behandelt, sondern nu als Randbedingung der gesellschaftlichen Selbstreproduktion.
} 
Augenfällig wird Integration also zunächst dort, wo sie schwindet, so daß plötzlich oder als Ergebnis eines anfangs unbemerkten schleichenden Prozesses entweder Desintegration oder Überintegration auftritt. Es gibt somit zwei Richtungen der Gefährdung gesellschaftlicher Integration: zu wenig oder zu viel Integration. Landläufig denkt man nur an ersteres im Sinne eines Zerfalls des gesellschaftlichen Ganzen, der gewissermaßen "von unten“, von dessen Teilen ausgeht. Aber auch Überintegration ist möglich: eine „von oben“ wirkende, das gesellschaftliche Leben gleichsam erstickende Einzwängung der Teile durch das Ganze, die dann auf dieses zurückschlägt. ${ }^{5}$

Integration wird, wie gerade gesagt, zum einen dann erfahren, wenn sie schwindet. Zum anderen kann aber auch das Schwinden von Des- bzw. Überintegration, also eine wieder erstarkende Integration als solche bewusst werden. Klar ist: Erst aus dem Vergleich mit der sich einstellenden oder wieder nachlassenden Abweichung kann man bei entsprechendem Bemühen erkennen, worauf Integration beruht; und auf die so in den Blick fallenden Kausalfaktoren und -zusammenhänge lässt sich dann möglicherweise auch gezielt einwirken, um zukünftig Integration erhalten oder wieder herstellen zu können. Doch sobald die Des- bzw. Überintegration abgewehrt oder beseitigt ist, rücken die entsprechenden Zusammenhänge leicht wieder in den Hintergrund, werden aufs neue für selbstverständlich genommen, bis die nächste Störung droht oder eintritt.

Versteht man gesellschaftliche Integration in diesem Sinne negativ als Abwesenheit von Des- oder Überintegration, kann man sich weiterhin den graduellen Charakter von Integration klar machen. Sie stellt eine Balance zwischen Des- und Überintegration dar, einen mittleren Ordnungszustand, der durch ein Mehr oder durch ein Weniger an Ordnung gestört werden kann. Gesellschaftliche Integration ist damit auch ein zeitpunkt-relativer Maßstab. Aussagen darüber machen nur so Sinn, dass der Gesellschaftszustand zum Zeitpunkt $\mathrm{t}_{1}$ integrierter beziehungsweise weniger integriert als zum Zeitpunkt to ist. Man verfügt also über keinen absoluten, außerhistorischen Maßstab für das Niveau gesellschaftlicher Integration. Gerade soziologische Gegenwartsdiagnosen brauchen einen solchen Maßstab aber auch gar nicht. Sie wollen ja nichts anderes, als das Heute mit dem Gestern und dem vorstellbaren Morgen zu $(\leftarrow$ p.4) vergleichen, um Aussagen über Krisentendenzen beziehungsweise sich eröffnende

Die Redeweise von Ganzem und Teilen sei trotz ihrer bekannten Mängel an dieser Stelle gestattet, um vom Thema abführende kompliziertere Erörterungen über den gesellschaftlichen Reproduktionszusammenhang zu vermeiden. 
historische Chancen der gesellschaftlichen Dynamik zu treffen. Es geht gar nicht um die Suche nach einer optimal integrierten, also diesbezüglich perfektionierten Gesellschaft. Sondern gefragt wird danach, ob die gesellschaftliche Integration heute besser oder schlechter ist als sie es gestern war, und was diesbezüglich für Zukunftsaussichten bestehen.

Ebenso wichtig wie das negative und das graduelle Integrationsverständnis ist für einen handhabbaren Maßstab eine weitere analytische Dekomposition dessen, was gesellschaftliche Integration umfasst. Was sind die anderen Einheiten, zu denen die Gesellschaft ein für ihre Selbstreproduktion essentielles Verhältnis unterhält? An diesem Punkt kann man sich die Entwicklung der soziologischen Gesellschaftstheorie ebenso wie die Parallelentwicklung soziologischer Zeitdiagnosen vor Augen führen. Beide Entwicklungen haben inzwischen - und dies ist bereits ein vorweg genommenes wichtiges Ergebnis der vergleichenden Analyse der hier betrachteten Gegenwartsdiagnosen zu einer dreidimensionalen Auffächerung des Verständnisses gesellschaftlicher Integration geführt.

Bis vor nicht allzu langer Zeit erfasste die von David Lockwood (1964) eingeführte Unterscheidung von Sozial- und Systemintegration alle wesentlichen Aspekte dessen, was unter gesellschaftlicher Integration verstanden wurde. ${ }^{6}$ Diese beiden Integrationsdimensionen bezeichnen gänzlich andersartige Bezugspunkte gesellschaftlicher Integration - oder anders ausgedrückt: Es werden zwei völlig verschiedene Störungsquellen gesellschaftlicher Integration herausgestellt. Unter dem Gesichtspunkt der Sozialintegration geht es um die Integration der einzelnen Gesellschaftsmitglieder als Personen in die Gesellschaft. Dies ist das klassische Thema des Verhältnisses von Individuum und Gesellschaft. Weder dürfen ungebändigte Persönlichkeiten die gesellschaftliche Ordnung ruinieren, noch darf eine zu restriktive Ordnung auf gesellschaftlich ebenso dysfunktionale Weise die Kreativität der Personen unterdrücken. Der Gesichtspunkt der Systemintegration stellt demgegenüber auf die Integration der verschiedenen Teilsysteme der modernen Gesellschaft in diese ab. Das bedeutet, auf ein bestimmtes Teilsystem bezogen: dessen Integration mit den jeweils anderen Teilsystemen. Die Teilsysteme - siehe z.B. das Verhältnis von Wirtschaft und Politik - dürfen

6 Die weitere Verwendung und Diskussion der beiden Konzepte hat allerdings gegenüber Lockwoods ursprünglichem Vorschlag nicht nur Klärungen, sondern auch neue Uneindeutigkeiten und Widersprüche hervorgebracht (Mouzelis 1997). Hier wird an die Verwendung beider Begriffe bei Luhmann (1997: 618/619) angeknüpft. 
einander wechselseitig keine unlösbaren Probleme bereiten. Hier steht also das intersystemische Interdependenzgeflecht in seinen Auswirkungen auf die teilsystemspezifischen Leistungsproduktionen im Blickpunkt. Weder darf ein Teilsystem durch andere Teilsysteme beispielsweise hinsichtlich benötigter Ressourcen im Stich gelassen werden, noch dürfen diese ihm, etwa durch Zwangsmaßnahmen oder Sachzwänge, seine Autonomie nehmen - um nur zwei mögliche Störungen der gesellschaftlichen Systemintegration anzuführen.

Mit der Unterscheidung von Sozial- und Systemintegration ließen sich die Aspekte gesellschaftlicher Integration, die bis in die siebziger Jahre hinein die Aufmerksamkeit beherrschten, zuordnen und systematisieren. Seitdem ist allerdings in den ge- $(\leftarrow$ p.5) sellschaftlichen Debatten und auch in soziologischen Zeitdiagnosen ein vorher gar nicht beachtetes oder allenfalls randständiges Thema prominent geworden: die ökologische Integration der modernen Gesellschaft, also das Verhältnis der Gesellschaft zu ihrer physikalischen, chemischen und biologischen Umwelt (Reese-Schäfer 1996: 381). ${ }^{7}$ Der erste Bericht des Club of Rome über die „Grenzen des Wachstums“ war die wichtigste Initialzündung. In den achtziger und neunziger Jahren hat die ökologische Integration in der Gesellschaftsbeobachtung eine gleichrangige Bedeutung wie die Sozial- und die Systemintegration gewonnen; zeitweise übertrumpften ökologische Besorgnisse und Ängste sogar alles andere. Insbesondere der Reaktorunfall von Tschernobyl wurde - nicht nur für Ulrich Becks (1986) Konzept der „Risikogesellschaft“ - zum symbolträchtigen Ereignis.

Ökologische Integration heißt: Die Reproduktionsfähigkeit der modernen Gesellschaft hängt entscheidend auch davon ab, dass sie sich innerhalb der von der Natur gesetzten Grenzen - beispielsweise des Energieverbrauchs - auf einem langfristig bestandssicheren Pfad bewegt. Diese Grenzen sind nicht starr, sondern können vor allem durch wissenschaftlich-technische Innovationen hinausgeschoben werden - wie weit, weiß allerdings niemand. ${ }^{8}$

\footnotetext{
${ }^{7}$ Mit Talcott Parsons könnte man sagen, daß der Gesellschaft als sozialem System die Belange ökologischer Integration über den Verhaltensorganismus der Gesellschaftsmitglieder vermittelt werden: Hunger, Frieren, Einschränkungen der Bewegungsfreiheit, Krankheiten u.ä. - schließlich Tod (Parsons/Platt 1973: 436).

${ }^{8}$ Siehe auch den profunden Überblick bei Berger (1994).
} 
Diese wenigen Andeutungen zu den drei Dimensionen gesellschaftlicher Integration müssen zunächst genügen, um ein Tableau zu skizzieren, in das Topoi der verschiedenen Gegenwartsdiagnosen eingeordnet werden können (siehe Abbildung 2).

Integrationsdimensionen (Abb. 2)

\begin{tabular}{c|l|l|l|} 
& Sozialintegration & Systemintegration & $\begin{array}{l}\text { ökologische In- } \\
\text { tegration }\end{array}$ \\
\cline { 2 - 4 } Desintegration & & & \\
\hline \multirow{2}{*}{ Integrationsprobleme } & & & \\
\cline { 2 - 4 } & & & \\
& & & \\
\cline { 2 - 4 } & & & \\
\end{tabular}

Die Dimensionen sind hier erst einmal unverbunden nebeneinander aufgeführt worden. Aber es liegt nahe, dass zwischen innen starke Wechselwirkungen bestehen. Auch darauf wird zu achten sein, wenn nun die Thesen der soziologischen Gegenwartsdiagnosen zur gesellschaftlichen Integration näher betrachtet werden. Wie sich zeigen wird, setzen die verschiedenen Diagnosen ganz unterschiedliche Akzente $1 \leftarrow$ p.6) hinsichtlich der drei Integrationsdimensionen und ihrer Verknüpfungen. Das Spektrum reicht von solchen Analysen, die - wie etwa James Colemans (1982) Überlegungen zur "asymmetrischen Gesellschaft“ - lediglich eine der drei Dimensionen ansprechen, und dies auch nur hinsichtlich eines ganz spezifischen Aspekts, bis hin zu Betrachtungen wie Peter Gross' (1994) Porträt der „Multioptionsgesellschaft", das zumindest implizit das Zusammenspiel aller drei Integrationsdimensionen thematisiert.

Die Unterscheidung der drei Integrationsdimensionen dient im Weiteren also als erstes grobes Sortierschema für zentrale Aussagen der betrachteten soziologischen Gegenwartsdiagnosen. Zunächst wird die Systemintegration, dann die ökologische Integration und schließlich die Sozialintegration betrachtet. 


\section{Systemintegration}

Im Hinblick auf die Systemintegration stellt sich die gesellschaftliche Lage für die soziologischen Zeitdiagnosen schon seit längerem so dar, dass keine wirklich kritischen Probleme drohen. Dies wird nicht einmal explizit festgestellt, sondern dokumentiert sich vor allem darin, dass die soziologischen Gegenwartsdiagnosen der zurückliegenden zwanzig Jahre systemintegrative Probleme nicht an prominenter Stelle diskutieren.

Vor dem Hintergrund der soziologischen Zeitdiagnosen aus dem letzten Jahrhundert versteht sich diese Entwarnung keineswegs von selbst. Damals wurde bekanntlich die fortschreitende Ausdifferenzierung autonomisierter Teilsysteme für Wirtschaft, Wissenschaft, Kunst, Massenmedien und weitere "Wertsphären" - um Max Webers Begrifflichkeit aufzugreifen - höchst misstrauisch betrachtet. So thematisierte etwa Karl Marx die Verselbständigung der kapitalistischen Wirtschaft als einen Vorgang, der einen katastrophalen Zusammenbruch gesellschaftlicher Systemintegration nach sich ziehen würde. Gerade die teilsystemisch höchst erfolgreiche "Akkumulation des Kapitals“ in Form einer „Reproduktion auf erweiterter Stufenleiter" (Marx 1867: 605-617), also - in Niklas Luhmanns (1984) Terminologie - die Autopoiesis der Zahlungen werde der Gesellschaft als Ganzer zum Verhängnis. Karl Polanyi (1944) griff diese Vorstellungen noch in den vierziger Jahren dieses Jahrhunderts wieder auf. Ein Wirtschaftssystem, das weitestgehend aus allen anderen gesellschaftlichen Bezügen "entbettet" sei, stelle einen permanenten Krisenherd für die gesellschaftliche Ordnung dar. Zerrüttung der Familien und politische Instabilität wurden als die gravierendsten negativen Auswirkungen der kapitalistischen Wirtschaft auf andere gesellschaftliche Teilsysteme thematisiert. Darüber hinaus wurden aber auch immer wieder $(\leftarrow$ p.7) negative Folgewirkungen auf das Bildungs-, das Gesundheits-, das Wissenschafts- und das Religionssystem angesprochen.

Max Weber (1919: 27/28) relativierte Marx' Diagnose einer tiefgreifenden systemintegrativen Krise der modernen Gesellschaft und dramatisierte diese Diagnose zugleich mit seiner These des "Polytheismus" der "Wertsphären". Für Weber ist es nicht nur die 
kapitalistische Ökonomie, die sich durch Ausdifferenzierung autonomisiert und damit für unzuständig hinsichtlich aller sonstigen gesellschaftlichen Belange erklärt. Bei allen anderen gesellschaftlichen Teilsystemen lässt sich gleiches beobachten. So kümmert sich beispielsweise die Wissenschaft im sprichwörtlichen Elfenbeinturm nicht mehr um die gesellschaftliche Nützlichkeit oder auch Gefährlichkeit ihrer Erkenntnisse; und ähnlich unbekümmert schürt ein allein auf "newsworthiness" fixiertes System der Massenmedien durch extensive Skandalberichterstattung "Politikverdrossenheit", die die Stabilität eines demokratischen politischen Systems gefährden kann. Webers Relativierung des von Marx ausschließlich betonten Kapitalismus als Gefährdung gesellschaftlicher Systemintegration läuft also nicht auf eine optimistischere Sicht der Dinge hinaus. Im Gegenteil diagnostiziert Weber eine multiple und damit entsprechend schwer beherrschbare Anfälligkeit der modernen Gesellschaft für systemintegrative Krisen. Die funktionale Differenzierung erzeugt vielfache zentrifugale Tendenzen und Zerrissenheiten der modernen Gesellschaft.

Heutzutage muten so angelegte Krisendiagnosen wenig überzeugend an. Nicht, dass die damaligen Verweise auf gesellschaftliche Bruchstellen und Katastrophenherde als gänzlich falsch oder zumindest heillos übertrieben hingestellt werden könnten! Doch ganz offensichtlich - so die heutige Sichtweise - hat die moderne Gesellschaft gelernt, wie sie ihre systemintegrativen Probleme in den Griff zu bekommen vermag. Zu diesem Lernen haben vermutlich auch soziologische Zeitdiagnosen beigetragen, deren warnende Stimmen als sich selbst widerlegende Prophezeiungen gewirkt haben. So ist bekanntlich der Sozialstaat, der die kapitalistische Wirtschaft wieder "gesellschaftsfähig" gemacht hat, nicht zuletzt dadurch inspiriert worden, dass die politischen Interessenvertreter sowohl der Arbeiterschaft als auch der "Kapitalisten" ihre Lehren aus Marx gezogen haben. ${ }^{9}$

Der Sozialstaat ist nur das vielleicht prominenteste Beispiel dafür, wie ein systemintegrativer Brennpunkt des gesellschaftlichen Geschehens durch geeignete institutionelle Mechanismen so umgestaltet werden kann, dass vormalige Krisen in handhabbare Probleme kleingearbeitet worden sind. Auch andere Arten staatlicher Förderung gesellschaftlicher Systemintegration durch politische Gesellschaftssteuerung sind seit dem letzten Jahrhundert stetig institutionalisiert worden. So kümmert sich etwa die

\footnotetext{
${ }^{9}$ Siehe etwa die einflußreiche zeitdiagnostische Programmschrift von Eduard Heimann (1929).
} 
Bildungspolitik um die Abstimmung zwischen Bildungs- und Wirtschaftssystem, oder die Forschungspolitik sorgt dafür, dass sich die wissenschaftliche Forschung unter anderem auch an den Erfordernissen des Gesundheitssystems orientiert. Man kann ohne Übertreibung sagen, dass die Herausbildung des "Steuerungsstaates" (Kaufmann 1991) eine gesellschaftliche Reaktion auf vielfältige systemintegrative Probleme gewesen ist. Man darf sich dies freilich niemals als unmittelbare Bewirkung entsprechender Steuerungsmaßnahmen durch die jeweils objektiv gegebenen Prob- $(\leftarrow \mathbf{p} . \mathbf{8})$ leme vorstellen. Erst wenn diese Probleme durchsetzungsfähige politische Träger, etwa Interessengruppen, finden und es weiterhin auch geteilte Problemdeutungen - an denen zunehmend professionelle Experten arbeiten - gibt, können systemintegrative Probleme ihre politische Bearbeitung finden.

Nur der kleinere Teil systemintegrativer Probleme der modernen Gesellschaft wird allerdings politisch bearbeitet. Die meisten systemintegrativen Probleme werden im Vorfeld einer Politisierung durch direkte Abstimmungen zwischen gesellschaftlichen Teilsystemen bewältigt. Dies haben aus der Perspektive der Theorien gesellschaftlicher Differenzierung sowohl Talcott Parsons mit seinem Konzept der "double interchanges" als auch Luhmann mit dem Verweis auf "strukturelle Kopplungen" zwischen den Teilsystemen herausgearbeitet. ${ }^{10}$ Wenn zum Beispiel Professoren an Hochschulen Forschungsgelder von Unternehmen erhalten, ist dies ein - von beiden Akteuren zumeist so nicht intendierter - Beitrag zur Integration von Wissenschafts- und Wirtschaftssystem. Denn es werden Forschungsthemen gefördert, die versprechen, die Innovationsfähigkeit und damit Prosperität der Wirtschaft zu verbessern. Unzählige dezentrale Abstimmungen dieser Art finden insbesondere zwischen Organisationen verschiedener gesellschaftlicher Teilsysteme permanent statt, so dass man interorganisatorische Beziehungen und Netzwerke, die Teilsystemgrenzen überschreiten, als wichtige Garanten gesellschaftlicher Systemintegration ansehen kann. Nur wo derartige direkte und dezentrale Abstimmungen zwischen den Teilsystemen versagen, wird überhaupt politische Gesellschaftssteuerung auf den Plan gerufen. Sie kann sich dann, wie schon angesprochen, zum einen durch eigene Gestaltungsmaßnahmen in die jeweilige systemintegrative Problematik einschalten. Zum anderen beschränken sich staatliche Akteure aber häufig auch auf eine "prozedurale Steuerung" (Offe 1975) derart, dass Verhandlungen zwischen Repräsentanten der betreffenden Teilsysteme ins Leben

10 Das folgende basiert auf der ausführlichen Darstellung bei Schimank (1999). 
gerufen oder gefördert werden, also wiederum die Befähigung zur direkten Abstimmung zwischen den Teilsystemen gestärkt wird.

Richard Münchs (1991) gegen Luhmann zugespitzte These, daß „Interpenetration“ der verschiedenen Teilsysteme, also die wechselseitige Durchdringung der Teilsystemlogiken, die moderne Gesellschaft charakterisiere und sogar noch immer stärker um sich greife, erhebt diese Bearbeitungsmechanismen systemintegrativer Probleme geradezu zum Wesensmerkmal der westlichen Moderne - womit Münch am unmißverständlichsten von allen soziologischen Gegenwartsbeobachtern ausdrückt, daß Systemintegration nicht die Integrationsdimension ist, die zur Zeit als prekär angesehen wird. Mit all dem, was politikwissenschaftliche Betrachtungen politischer Gesellschaftssteuerung und die Analyse intersystemischer Beziehungen durch die soziologische Differenzierungstheorie an empirischen und theoretischen Befunden zusammengetragen haben, ist zwar nicht gesagt, dass die Systemintegration der modernen Gesellschaft nunmehr ein für alle Male gesichert, eine krisenhafte Zuspitzung also dauerhaft ausgeschlossen sei. Es gibt bislang allerdings kaum Anzeichen dafür.

Zwei Ausnahmen müssen erwähnt werden, die beide in modifizierter Form die Marx'sche Kapitalismuskritik wieder aufgreifen, also aufs Neue gravierende Probleme thematisieren, die das Wirtschaftssystem anderen Teilsystemen bereitet. Pierre $\leftarrow \leftarrow$ p.9) Bourdieu (1998) sieht eine "Intrusion" wirtschaftlicher Kräfte und Gesichtspunkte in verschiedene "kulturelle" Teilsysteme. Kunst, Bildung, Wissenschaft und Massenmedien werden demnach immer stärker kommerziellen Erwägungen der Gewinnerzielung sowie Gesichtspunkten der wirtschaftlichen Nützlichkeit ihrer Leistungen unterworfen; und in gleichem Maße schwindet die jeweilige teilsystemische Autonomie. Für Bourdieu sind diese Tendenzen Ausdruck davon, dass politische Gesellschaftssteuerung die betreffenden anderen Teilsysteme nicht länger vor einer auch durch die "Globalisierung" entfesselten kapitalistischen Wirtschaft schützt. Ähnlich argumentiert Richard Sennett (1998) hinsichtlich der negativen Effekte einer durch "Globalisierung" und "neoliberale" Wirtschaftspolitik in Verbindung mit den neuen Informations- und Kommunikationstechnologien "flexibilisierten" Wirtschaft für das Familiensystem. Steigende Scheidungsraten, Verzicht auf dauerhafte Partnerbeziehungen und auf Kinder sowie alle Arten von zerrütteten Familienverhältnissen und Erziehungsdefiziten sind demzufolge in erheblichem Maße dadurch bedingt, dass ein "flexibler Kapitalismus" 
entsprechend "flexible Menschen" erfordert, die sich langfristige zwischenmenschliche Bindungen in Gestalt von Familien zunehmend weniger leisten können. Weder Bourdieu noch Sennett würden allerdings in diesen negativen Externalitäten des Wirtschaftssystems für die betreffenden anderen Teilsysteme bislang systemintegrative Gefährdungen der modernen Gesellschaft insgesamt erkennen. Dies zeigt sich nicht zuletzt darin, dass beide Autoren die von innen diagnostizierten Tatbestände moralisch kritisieren, diese also nicht funktionalistisch als gefährliche Beeinträchtigung der Reproduktionsfähigkeit der modernen Gesellschaft einstufen.

Alles bisher zur gesellschaftlichen Systemintegration Gesagte unterstellt, dass in dieser Dimension Desintegration die zentrale Art von Problem ist. Demgegenüber gibt Luhmann (1997: 617/618) andeutungsweise zu bedenken, ob die heutige Gesellschaft nicht eher zu einer problematischen Überintegration tendiere. Dies könnte sogar - so ließe sich Luhmann weiterdenken - die längerfristige Folgewirkung einer über ihr Ziel hinaus schießenden Bewältigung von Problemen der Desintegration zwischen gesellschaftlichen Teilsystemen sein. Dass die Teilsysteme der modernen Gesellschaft sich verselbständigen, rücksichtslos miteinander umgehen und einander dabei immer wieder in die Quere kommen, ist ein Auswuchs der für die Leistungsfähigkeit der Teilsysteme konstitutiven Autonomie. Dem mit Blick auf die gesellschaftliche Systemintegration entgegen zu wirken, kann sich schnell seinerseits zur Autonomiegefährdung auswachsen.

Das eindringlichste, erst vor kurzer Zeit historisch gewordene Beispiel für eine Überintegration stellten die sozialistischen Gesellschaften Osteuropas dar. Dies waren - um einen auf Frankreich gemünzten Ausdruck Michel Croziers (1970) aufzugreifen - "blockierte Gesellschaften" in dem Sinne, dass der hypertrophe Anspruch umfassender politischer Gesellschaftssteuerung alle gesellschaftlichen Teilsysteme so eng miteinander verzahnte und unter politische Kuratel stellte, dass die Effizienz und Innovationskraft der teilsystemischen Leistungsproduktionen irreparablen Schaden nahm. Weit weniger dramatische Fälle der Überintegration lassen sich aber auch in nichtsozialistischen Gesellschaften finden. Um ein bereits benutztes Beispiel fortzuführen: Wenn die Forschungen von Professoren zumindest in bestimmten Wissenschaftsgebieten zu stark von Geldern aus der Industrie abhängig werden, könnte $(\leftarrow \mathbf{p} .10)$ dies mittelfristig sowohl für das Wissenschafts- als auch für das Wirtschaftssystem ein 
gravierendes Problem erzeugen. Die Wissenschaft verzettelte sich in kurzfristigen Anwendungsbezügen und vernachlässigte die Grundlagenforschung, und dies beeinträchtigte dann in der Folgewirkung auch die technologische Innovationsfähigkeit der Wirtschaft. Das Beispiel zeigt freilich auch, dass schon einiges geschehen müsste, bis eine derartige zu enge Kopplung zwischen zwei Teilsystemen sich tatsächlich krisenhaft zuspitzt. Viel wahrscheinlicher ist, dass es vorher Alarmreaktionen und Widerstand von Seiten der Betroffenen, ihrer Interessengruppen oder weitsichtiger politischer Akteure gibt.

Zusammenfassend lässt sich also festhalten, dass die gesellschaftliche Systemintegration von den soziologischen Gegenwartsdiagnosen größtenteils nicht thematisiert wird. Hier werden mögliche oder gar für wahrscheinlich gehaltene Krisentendenzen der heutigen Gesellschaft also nicht ausgemacht. Das ist, um es noch einmal zu wiederholen, ein markanter Unterschied gegenüber Zeitdiagnosen, wie sie im neunzehnten Jahrhundert und auch noch in den ersten Jahrzehnten des zwanzigsten Jahrhunderts vorgelegt wurden. Die Abstimmung zwischen den gesellschaftlichen Teilsystemen funktioniert also im Großen und Ganzen so, dass keine größeren und dauerhaften Reibungsflächen entstehen. Die heute und, so wird prognostiziert, erst recht morgen für die Zukunftsfähigkeit der modernen Gesellschaft entscheidenden Integrationsprobleme müssen somit in den anderen beiden Integrationsdimensionen lokalisiert werden.

\section{Ökologische Integration}

Zunächst sei die ökologische Integration der modernen Gesellschaft angesprochen. Anfang der siebziger Jahre erwähnte Jürgen Habermas (1973: 61-63) in seiner damaligen Zeitdiagnose ökologische Krisenpotentiale "spätkapitalistischer" Gesellschaften zwar bereits kurz, ging aber noch ganz selbstverständlich davon aus, dass dies jedenfalls nicht die Schicksalsfrage der modernen Gesellschaft sein würde. Das Problem der ökologischen Integration der modernen Gesellschaft war damals also noch ähnlich latent, wie es das Problem ihrer Systemintegration heute ist.

Die Art und Weise, wie die moderne Gesellschaft seitdem auf ihre ökologische Integration aufmerksam geworden ist, ist ein Musterbeispiel für das Zusammenspiel von 
objektiv gegebenen Problemlagen, professionellen Problemdeutungen und gesellschaftlichen Akteuren, die sich in ihren Interessen betroffen vorkommen. Ob es um Energieversorgung oder Nahrungsmittelproduktion, um Umweltgift oder Klimaveränderungen, um Müllbeseitigung oder Artenvielfalt geht: Stets ist - wie insbesondere Ulrich Beck (1986) immer wieder herausstellt - Expertenwissen nötig, das die jeweiligen Probleme überhaupt erst sichtbar macht; und nur sofern dieses Expertenwissen insbesondere über die Massenmedien vermittelt in die allgemeine gesellschaftliche Kommunikation und die teilsystemspezifischen Kommunikationszusammenhänge eingeht, ist die ökologische Integration - wie Luhmann (1986: 63) ganz dezidiert betont - ein gesellschaftliches Problem. Die Gesellschaft könnte sich ökolo- $(\leftarrow$ p.11) gisch völlig ruinieren: So lange sie sich dies nicht kommunikativ bewusst macht, geht sie zu Grunde, ohne zu merken, woran.

Eine bis zu den tiefsten Wurzeln des Selbstverständnisses der Moderne vorstoßende Analyse des Problems der ökologischen Integration stammt von Bruno Latour (1991). Für inn unterliegt die moderne Gesellschaft einer fundamentalen Selbsttäuschung, die zunächst für eine lange Erfolgsgeschichte wissenschaftlich-technischer Innovationen gesorgt hat, seit einiger Zeit dann allerdings immer stärker ihre Schattenseite in Gestalt von ökologischen Problemen gezeigt hat. Die Selbsttäuschung beruht darin, dass die moderne Weltdeutung auf einer strikten ontologischen Trennung von Natur auf der einen und Sozialwelt auf der anderen Seite beruht, so dass naturwissenschaftliche Erkenntnisse und technische Artefakte als "Hybride" eine immer größer werdende Zwischenwelt bevölkern. Diese Zwischenwelt wird aber eben nicht als solche anerkannt, sondern künstlich in soziale und natürliche Bestandteile aufgespalten. Genau dadurch nimmt sich die moderne Gesellschaft selbst die Chance, ihre Erzeugung und Verbreitung der "Hybride" unter Kontrolle zu halten. Die Moderne praktiziert für Latour eine naturwüchsige Vergesellschaftung der Natur. Das hat dazu geführt, daß sich dieser Vorgang enorm ausweiten konnte - beispielsweise weil traditionelle normative Beschränkungen dessen, was man technisch „machen“ darf, durch die kollektive Selbsttäuschung überwunden werden konnten. Doch die ungezügelte Vermehrung der „Hybriden“ führt zu einer allmählichen Verschiebung der Kosten/Nutzen-Bilanz. Denn je weiter die „Netze“ der Innovationen gespannt werden, desto mehr verfangen sich die verschiedenen „Netze“ auch ineinander, woraus dann immer mehr ungeahnte Neben- 
und Fernwirkungen resultieren - und vieles Ungeahnte ist dann auch mehr oder weniger unerwünscht und nicht selten ausgesprochen gefährlich.

Während Latour somit die gestörte ökologische Integration der modernen Gesellschaft auf eine substantielle ontologische Prämisse der modernen Kultur zurückführt, heben sowohl Beck als auch Luhmann das gesellschaftliche Formprinzip der funktionalen Differenzierung als zentrale Ursache der vorfindbaren Desintegrationstendenzen hervor. Für Beck (1986) ist die moderne Gesellschaft in ökologischer Hinsicht eine "Risikogesellschaft", weil diesbezüglich wirtschaftliche, wissenschaftliche und politische Kräfte in "organisierter Unverantwortlichkeit" zusammenwirken. Alle drei gesellschaftlichen Teilsysteme folgen je eigenen Handlungslogiken, die sie strukturell blind für ökologische Belange machen und auch dafür sorgen, dass diese Blindheiten einander nicht wenigstens wechselseitig korrigieren. Wirtschaftliches Gewinn-, politisches Macht- und wissenschaftliches Wahrheitsstreben sind gleichermaßen allenfalls zufällig an einem "nachhaltigen" Verhältnis der modernen Gesellschaft zu ihrer natürlichen Umwelt interessiert. Luhmann (1986) gelangt zu einem sehr ähnlichen Ergebnis, generalisiert es jedoch noch auf alle übrigen gesellschaftlichen Teilsysteme. Auch beispielsweise das Recht, die Religion, das Bildungswesen oder die Massenmedien haben in ihren jeweiligen binären Codes, die die teilsystemischen Kommunikationszusammenhänge prägen, keine Resonanz für ökologische Belange vorgesehen. Damit ist zunächst einmal in jedem Teilsystem eine hohe Schwelle der Thematisierbarkeit von Fragen ökologischer Integration gegeben; und selbst wenn solche Fragen angesprochen werden können, geschieht dies nicht so, dass ökologische $(\leftarrow$ p.12) "Nachhaltigkeit" zu einem wichtigen oder gar vorrangigen Gesichtspunkt teilsystemischen Operierens würde. In jedem Teilsystem bleibt die ökologische Integration der modernen Gesellschaft ein Aspekt, der neben vielen anderen steht und entsprechend mit innen um Aufmerksamkeit konkurriert. Das wird, was Luhmann ebenso wie Beck konstatiert, der mittlerweile gegebenen Virulenz der ökologischen Frage keineswegs gerecht.

Wer - wie Beck, und anders als Luhmann - eine akteurtheoretische Perspektive auf die gesellschaftliche Differenzierung benutzt, vermag auch herauszustellen, dass neben den ökologisch blinden teilsystemischen Codes Konkurrenzkonstellationen innerhalb der Teilsysteme zur Erzeugung und Verfestigung der ökologischen Probleme 
beitragen. Für ökologische "Nachhaltigkeit" erforderliche Selbstbeschränkungen der teilsystemischen Leistungsproduktion - wie sie auch Latour für wissenschaftlich-technische Innovationen anspricht - können nur äußerst schwer institutionalisiert werden, wenn Unternehmen um Marktanteile, Politiker um Wählerstimmen und Wissenschaftler um Reputation konkurrieren und dafür jeweils Leistungssteigerungen einsetzen. Im Zentrum der "organisierten Unverantwortlichkeit" stehen ineinander verzahnte wirtschaftliche, politische, militärische und wissenschaftliche Konkurrenzkonstellationen.

Verstreute Hinweise bei den genannten drei Autoren sowie bei Anthony Giddens (1990) systematisierend, kann man analytisch zwischen der Erzeugung, der Thematisierung und der Bearbeitung von Problemen der ökologischen Integration unterscheiden und die verschiedenen gesellschaftlichen Teilsysteme entsprechend zuordnen:

Problemerzeugung: Hier werden zunächst, wie schon erwähnt, das Wirtschaftsund das Wissenschaftssystem genannt. Weiterhin ist aber zunehmend, gerade angesichts des Einsatzes von ABC-Waffen, auch das Militärsystem involviert. Über ein immer weiter steigendes Konsumniveau trägt auch das Familiensystem - als Abnehmer des Wirtschaftssystems - zur Erzeugung ökologischer Probleme massiv bei. Noch weniger in den Blick geraten sind zwei weitere Teilsysteme, die jeweils auf ihre Weise - zusammen mit dem Familiensystem - für ein weltweit nach wie vor kaum gebremstes Bevölkerungswachstum sorgen: das Gesundheitssystem durch verschiedene Effekte des medizinischen Fortschritts sowie das Religionssystem durch in vielen Weltreligionen verbreitete, Maßnahmen der Geburtenkontrolle ablehnende moralische Vorstellungen über Sexualität und Familie.

- Problemthematisierung: Hier steht das System der Massenmedien im Vordergrund, das allerdings entsprechende Informationen von Seiten des Wissenschaftssystems benötigt. Neben den Massenmedien wirkt auch, wiederum durch wissenschaftliche Erkenntnisse gespeist, das Bildungssystem daran mit, die ökologische Integration der modernen Gesellschaft zum Thema öffentlicher Kommunikation zu machen. Wenn entsprechende Kommunikationen nicht an jedes einzelne Gesellschaftsmitglied gerichtet werden und individuelle Verhaltensänderungen nahe legen, ist zumeist das politische System Adressat. Von inm wird kollektive Problembearbeitung erwartet. $(\leftarrow \mathbf{p . 1 3})$ 
- Problembearbeitung: Neben dem gerade erwähnten politischen System spielt hier das Rechtssystem eine wichtige Rolle. Es setzt politisch beschlossene regulative Maßnahmen zu Gunsten der ökologischen Integration um. Darüber hinaus trägt auch das Wirtschaftssystem dann zur Problembearbeitung bei, wenn dies lukrativ zu werden verspricht. Alle drei Teilsysteme sind freilich in ihrem entsprechenden Handeln wiederum stark darauf angewiesen, vom Wissenschaftssystem das erforderliche Wissen, insbesondere umgesetzt in Gestalt von Technologien, vermittelt zu bekommen.

Außer dem Sport- und dem Kunstsystem, die beide in ökologischer Hinsicht nur marginale Bedeutung haben, sind somit sämtliche Teilsysteme der modernen Gesellschaft auf die eine oder andere Weise in deren ökologische Integration involviert. Besonders hervorzuheben ist das Wissenschaftssystem, das an der Erzeugung, der Thematisierung und der Bearbeitung ökologischer Probleme gleichermaßen stark beteiligt ist.

Befragt man die soziologischen Gegenwartsdiagnosen daraufhin, welche Mechanismen für eine Wiederherstellung der gestörten ökologischen Integration der modernen Gesellschaft sorgen könnten, wird fast einhellig auf entsprechende soziale Bewegungen verwiesen. Den gesellschaftlichen Teilsystemen, insbesondere auch den etablierten Parteien und Verbänden im politischen System, wird nicht zugetraut, von sich aus und aus sich heraus etwas Nennenswertes für die ökologische Integration zu tun. $\mathrm{Zu}$ sehr bleiben die Teilsysteme auf ihre jeweiligen Logiken fixiert. Beck (1986) spricht denn auch von "Subpolitik", um damit zum Ausdruck zu bringen, dass die Ökologiebewegung zwar einerseits in dem Sinne politisch ist, das es hier um eine kollektive Gestaltung des gesellschaftlichen Naturverhältnisses geht - andererseits dürfe dies nicht einer ansonsten im politischen System etablierten Logik selbstzweckhaft verabsolutierter Machterhaltung und -steigerung folgen. Wie vor allem die Entwicklung Grüner Parteien zeigt, sind freilich zumindest wichtige Teile der "Subpolitik" keineswegs immun dagegen, den - um eine Marx'sche Terminologie zu übertragen - ökologischen "Gebrauchswert" ihrer Aktivitäten zu Gunsten eines machtpolitischen "Tauschwerts" aus den Augen zu verlieren. 
Luhmann (1986: 227-248) gibt noch grundsätzlicher zu bedenken, dass die Ökologiebewegung zwar auf der einen Seite zweifellos den Finger auf eine immer schmerzhafter klaffende Wunde der funktional differenzierten Gesellschaft gelegt habe, doch mehr als "Angstkommunikation", also Panikmache, die zwischen hilflosem Aktionismus und resignativer Lähmung oszilliert, brächten die sozialen Bewegungen nicht zu Stande. Von daher sieht Luhmann sie auch nicht als funktionstüchtige Mechanismen einer Wiederherstellung der ökologischen Integration. In seiner Theorieperspektive läge es näher, danach Ausschau zu halten, ob nicht möglicherweise für die Bearbeitung ökologischer Probleme ein neues gesellschaftliches Teilsystem im Werden begriffen sein könnte. Dessen Keimzellen könnten - wie es in der Frühphase anderer Teilsysteme ebenso der Fall war - bislang noch über diverse existierende Teilsysteme verstreut sein. Bürgerinitiativen könnten ebenso dazu gehören wie Ansätze einer ökologisch orientierten Wissenschaft, einer entsprechend spe- $(\leftarrow \mathbf{p}$.14) zialisierten massenmedialen Öffentlichkeitsarbeit, Greenpeace oder auf ökologische Belange ausgerichtete Zweige der Wirtschaft. In dem Maße, wie diese sehr heterogenen kollektiven und korporativen Akteure ihr Handeln vor allen sonstigen Gesichtspunkten an einem gemeinsamen Code ökologischer "Nachhaltigkeit" orientierten, fänden sie sich zu einem Teilsystem zusammen; und wenn dieses ökologieorientierte Teilsystem dann irgendwann eine gewichtige Stimme im Konzert aller gesellschaftlichen Teilsysteme besäße, hätte die moderne Gesellschaft ihr Problem der ökologischen Integration gewissermaßen systemintegrativ "internalisiert", nämlich zu einem Abstimmungserfordernis zwischen diesem neuen Teilsystem und den übrigen Teilsystemen gemacht.

Eine solche, hier und heute natürlich nur hochgradig spekulativ erörterbare Weise des gesellschaftlichen Umgangs mit dem Erfordernis ökologischer Integration besäße vermutlich gegenüber einem zu großen Vertrauen in soziale Bewegungen auch den Vorteil, dass existierende Desintegration nicht irgendwann in Überintegration umschlägt. Überintegration in der ökologischen Dimension hieße, dass die moderne Gesellschaft zu viel Rücksicht auf Naturgegebenheiten nimmt. Der stark angstbestimmten und entsprechend moralisierenden Kommunikationsform sozialer Bewegungen liegt ein derartiger ökologischer Fundamentalismus durchaus nahe. Er würde alle Eingriffe in Naturgegebenheiten ablehnen, sofern es auch nur vage vorstellbare neue Risiken gibt. Einige Züge aktueller Debatten über Gentechnik werden durchaus von einem solchen pauschal bleibenden Skeptizismus getragen, der als universelles Totschlagargument 
herhalten kann, wo immer man ihn einsetzen will. ${ }^{11}$ Solche wenig konstruktiven Kommunikationsmuster durch die nüchternere Logik eines gesellschaftlichen Teilsystems zu ersetzen, dürfte für eine effektive Bewältigung der Probleme ökologischer Integration nur förderlich sein.

Insgesamt bleibt somit zur ökologischen Integration festzuhalten, dass sie in mehreren soziologischen Gegenwartsdiagnosen in den Mittelpunkt gestellt und in einigen anderen zumindest mit angesprochen wird. Der gesellschaftlichen Konjunktur dieses Themas wird also soziologisch Rechnung getragen.

\section{Sozialintegration}

Diejenige Integrationsdimension, die seit den soziologischen Klassikern bis heute die meiste und differenzierteste Aufmerksamkeit soziologischer Zeitdiagnosen gefunden hat, ist die der Sozialintegration. Eine Gefährdung der gesellschaftlichen Sozialintegration ist dann gegeben, wenn die Individuen massenhaft - jeder für sich oder im konzertierten Zusammenwirken - auf die eine oder andere Weise anomisch handeln. Anomie kann - in lockerer Anknüpfung an Robert K. Merton (1949) - vor allem dreierlei Gestalt annehmen: harte, insbesondere auch gewaltsam ausgetragene Interessen- und Identitätskonflikte zwischen gesellschaftlichen Gruppen; individuelle Devianz gegenüber institutionalisierten Normen, etwa in Form von Kriminalität, einschließlich der fatalistischen Hinnahme dieser Devianz, z.B. als „Wegschau“- $(\leftarrow$ p.15) Gesellschaft; ${ }^{12}$ und individuelle Entfremdung im Sinne einer fehlenden subjektiven Plausibilität kultureller Sinnangebote.

Eine grobe Sortierung möglicher sozialintegrativer Probleme kann zum einen wiederum Des- und Überintegration unterscheiden.Bei den Desintegrationstendenzen lässt sich zunächst konstatieren, dass mittlerweile kaum noch jemand den von Luhmann (1984: 362) bissig kommentierten „einstimmig-monotonen Gesang vom Sinnverlust“ anstimmt. Dieser Topos war in vielen älteren soziologischen Zeitdiagnosen sehr

11 Nicholas Reschers (1980) allgemeine philosophische Betrachtungen des Skeptizismus weisen eindringlich auf diese Gefahren für eine sachlich orientierte Debatte hin.

12 Die Nichtahndung beobachteter Normverstöße als sozusagen „tätige Unterlassung“ bei der Reproduktion bzw. Erosion institutioneller Ordnungen betont Axelrod (1986) in seiner Modellierung des „norms game“ besonders. 
prominent. ${ }^{13}$ In der kosmologischen „Unbehaustheit“ einer säkularisierten und unüberschaubar komplexen Welt finde der einzelne immer weniger überzeugende Antworten auf die existentiellen Grundfragen, wie er leben wolle und warum das Leben sich überhaupt lohne. Weil es keine letztlich religiös fundierte alles übergreifende sinnstiftende Deutung der Welt mehr gebe, gerate das Individuum in eine tiefgreifende Identitätskrise. Derartige kulturpessimistische Einschätzungen der Moderne müssen schlicht als auf der ganzen Linie empirisch widerlegt abgebucht werden. Weder haben sich massenhaft Selbstmorde aufgrund existentieller Verzweiflung ereignet, noch kann man aus irgendwelchen anderen Indikatoren herauslesen, dass die individuelle Lebenszufriedenheit im weitesten Sinne bei größeren Teilen der Bevölkerung rapide und unter ein psychisch erträgliches Maß gesunken sei. Damit ist nicht bestritten, dass die Moderne dem einzelnen ganz spezifische und in diesen Hinsichten schwierigere Aufgaben der Identitätsfindung und -behauptung auferlegt als vormoderne Gesellschaften (Schimank 1985). Doch dass immer mehr Gesellschaftsmitglieder an diesen Aufgaben scheitern, trifft offensichtlich nicht zu.

Fragt man danach, wie die Personen heutzutage eine subjektiv sinnhafte Identität konstruieren, lautet die eine, vor allem von Luhmann (1984: 362-367; 1987) gegebene Antwort: Die Individuen definieren sich selbst durch Ansprüche, die sie an verschiedene gesellschaftliche Teilsysteme richten, und erfahren Selbstbestätigung und Selbstverwirklichung vor allem aus der Realisierung dieser Ansprüche. Wirtschaftlich vermittelte Konsum- und Freizeiterlebnisse, Gesundheitsleistungen, Kulturangebote und Unterhaltung durch Kunst und die Massenmedien, Intimität in Familie und Freundeskreis, Bildungschancen, Sporttreiben und -zuschauen: Dies sind teilsystemisch bereitgestellte Leistungen, die der moderne Anspruchsindividualismus auf immer höherem Niveau einfordert, wie vor allem Peter Gross in seinem Konzept der „Multioptionsgesellschaft" festhält. Immer mehr von allem, was die gesellschaftlichen Teilsysteme an vielfältigsten Optionen der Lebensführung offerieren: Dieses Identitätsmuster beendet den religiös begründeten Bedürfnisaufschub in Gestalt der Hoffnungen auf ein jenseitiges Paradies und verlangt sozusagen „paradise now!“ Nicht alle, aber viele dieser Ansprüche richten sich auf „schöne Erlebnisse“ im Sinne Gerhard Schulzes (1993). Auch die „Erlebnisgesellschaft" ist also eine Folge des Anspruchsindividualismus.

13 Siehe nur etwa noch die These vom „homeless mind“ bei Peter Berger et al. (1973). 
Sozialintegrativ funktioniert der Anspruchsindividualismus; Sinnkrisen als individuelle Entfremdungserscheinungen kommen nicht ernstlich auf. Luhmann (1983) weist je-( $\leftarrow$ p.16) doch darauf hin, dass sich aus dem Wirken dieses sozialintegrativen Mechanismus längerfristig ein systemintegratives Problem in Gestalt von „Anspruchsinflationen“ ergeben könnte. Verschiedene Teilsysteme könnten in ihrer Leistungsproduktion chronisch überfordert werden und darauf u.a. dadurch reagieren, dass sie immer mehr Ressourcen beanspruchen, die dann anderen Teilsystemen fehlen. Die „Kostenexplosion" im Gesundheitssystem ist ein Beispiel dafür; aber auch etwa das Bildungssystem könnte immer höhere Finanzmittel beanspruchen, was dann für intersystemische Verteilungskämpfe sorgen könnte. Noch gravierender wirken sich „Anspruchsinflationen“ und Leistungsüberforderungen aus, wenn es um natürliche Ressourcen geht. Das Wirtschaftssystem geht z.B. auf Konsumansprüche mit einem immensen Verbrauch nicht erneuerbarer Energien ein, was eine wichtige Ursache der ökologischen Desintegration der heutigen Gesellschaft ist. Damit werden Wechselwirkungen zwischen den drei Integrationsdimensionen erkennbar. Ein sozialintegrativ funktionstüchtiger Mechanismus hat dysfunktionale Folgewirkungen für die Systemintegration und ökologische Integration der modernen Gesellschaft.

Ansprüche beruhen mangels absoluter Maßstäbe für das, was als gerechtfertigt und erforderlich erscheint, auf Vergleichen. Zum einen wird hierzu die Zeitdimension herangezogen und „Fortschritt" angemahnt: Was gestern reichte, ist - allein schon deshalb! - heute ungenügend. Zum anderen bietet die Sozialdimension Vergleichsmöglichkeiten: Andere haben mehr. ${ }^{14}$ Damit kommen potentiell desintegrative Verteilungskonflikte angesichts sozialer Ungleichheit in den Blick.

Ubiquitäre soziale Ungleichheit stellt in der modernen Gesellschaft mit ihrer kulturell verankerten Gleichheitsnorm gewissermaßen eine unerschöpfliche Gelegenheitsstruktur für Anspruchshaltungen bereit: Dass andere mehr Optionen der Lebensführung - Einkommen, Wohnqualität, Bildungschancen, gesundheitliche Versorgung u.a.m. - besitzen, wird als ungerecht angesehen und muss daher beseitigt werden, wobei nicht an eine Nivellierung "nach unten“, sondern "nach oben“ gedacht wird. Eine Legitimierung von Ungleichheit kann in der Moderne letztlich nur über differentielle Leistung erfolgen; und dies wird immer schwieriger, weil zum einen das

14 Dass meistens wiederum andere weniger haben als man selbst, wird hingegen geflissentlich übersehen. 
Leistungsethos immer mehr zugunsten eines Ethos des Sich-auslebens an Boden verliert und zum anderen bei vielen Ungleichheiten immer deutlicher wird, dass sie weniger auf individuelle Unterschiede der Leistungsbereitschaft und -fähigkeit als vielmehr auf Faktoren wie „Beziehungen“ und „Glück“ zurückgehen. Wenn somit aus als illegitim angesehenen sozialen Ungleichheiten auf Seiten der Schlechtergestellten unabweisbare - also nicht etwa durch Machtausübung unterdrückbare - Ansprüche auf Besserstellung erwachsen, werden die Bessergestellten in diesem Konflikt über die Verteilung bestimmter teilsystemischer Leistungen dazu neigen, anstelle von Umverteilung auf Wachstum zu setzen, um selbst nichts abgeben zu müssen. Dann werden eben z.B. ehemalige Luxusgüter wie Fernreisen vielen zugänglich gemacht, frühere Privilegien in der medizinischen Versorgung zum allgemeinen Leistungsstandard erhoben oder Studienplätze aus dem Boden gestampft, damit nicht weniger talentierte Abkömmlinge der höheren Schichten zugunsten kluger Arbeiterkinder auf eine akademische Ausbildung verzichten müssen. Gross (1994: 337) bringt diese generelle $(\leftarrow \mathbf{p} .17)$ Steigerungsdynamik der „Multioptionsgesellschaft“ auf die lapidare Formel: „Alles allen!“.

Ungleichheiten bringen Anspruchsspiralen hervor, die in Verteilungskonflikte münden, die ihrerseits durch Wachstum der teilsystemischen Leistungsproduktionen zu entschärfen versucht werden. Was sozialintegrativ durchaus funktioniert, hat wiederum wie bereits oben erläutert - dysfunktionale Effekte auf die Systemintegration und die ökologische Integration der modernen Gesellschaft. Überdies wirkt dieser sozialintegrative Mechanismus keineswegs flächendeckend, sondern lässt einen mehr oder weniger großen Rest an Gesellschaftsmitgliedern unberücksichtigt. Sie werden nicht in die Leistungsversorgung der verschiedenen Teilsysteme inkludiert, sondern mehr oder weniger weitgehend aus ihnen und damit letzten Endes aus der Gesellschaft insgesamt exkludiert. Dahrendorf (1992), das Forschungsteam von Bourdieu (1993) sowie Luhmann (1997: 618-634) und der Kreis um Heitmeyer (1997a; 1997b) sprechen diese besondere Zuspitzung sozialer Ungleichheit an, die darin mündet, dass ein Teil der Bevölkerung als gesellschaftlich überflüssig angesehen wird. Dies sind nach verbreiteter Einschätzung auf Seiten der Inkludierten bloße „Kostgänger“, um nicht zu sagen „Schmarotzer“, deren Anteil an den teilsystemischen Leistungen - und Anspruch auf diese - möglichst gering gehalten werden muss. Größere Bevölkerungsteile in der Dritten Welt, aber auch die Bewohner von Ghettos und Slums in den reichen westlichen Ländern stellen Anschauungsmaterial dar, an dem sich studieren läßt, wie sich 
eine kumulative Exklusion aus immer mehr gesellschaftlichen Teilsystemen vollzieht, wodurch sukzessiv immer größere Streichungen bei den individuellen Optionspaletten erfolgen. Arbeitslosigkeit heißt dann eben nicht nur, dass Konsum und Freizeitaktivitäten stark eingeschränkt werden, sondern dass etwa auch der Zugang zu Gesundheitsleistungen, Massenmedien, Rechts- und Bildungswegen - letzteres vor allem auch für die Kinder des Betreffenden - erschwert oder gar ganz versperrt wird, was wiederum die Arbeitslosigkeit zementiert u.s.w.

Wie für soziale Ungleichheit allgemein gilt auch für derartige Exklusionserscheinungen: Eine systemintegrativ stabile funktional differenzierte Gesellschaft lässt diese sozialintegrativen Probleme gewissermaßen links liegen. Dass hieraus jedoch sowohl im Weltmaßstab als auch in vielen nationalen oder regionalen Kontexten Verteilungskonflikte erwachsen können, die dann oftmals gewaltsam explodieren, weil den Exkludierten gar keine anderen politischen Durchsetzungsmöglichkeiten verfügbar sind, ist hinreichend bekannt. Eine weitere Zuspitzung von Exklusion könnte daher sozialintegrativ höchst brisant werden. Man könnte freilich auch, ähnlich wie bei der ökologischen Integration, darüber spekulieren, ob nicht schon ein auf Exklusionskorrektur spezialisiertes neues gesellschaftliches Teilsystem entsteht: das Hilfesystem, das sich vor allem aus den beiden Politikfeldern der nationalen Sozialhilfe und der internationalen Entwicklungshilfe zusammensetzt (Luhmann 1997: 633/634). Je effektiver dieses Teilsystem sich allerdings der Exklusionskorrektur widmet, desto mehr schalten sich die bislang Exkludierten in die geschilderten gesellschaftlichen Verteilungskonflikte ein, was die entsprechenden Probleme der Systemintegration und ökologischen Integration noch weiter zuspitzt. Immer unverkennbarer tritt also hervor, dass die gesellschaftliche Sozialintegration auf der einen Seite, die beiden anderen $(\leftarrow$ p.18) Integrationsdimensionen auf der anderen Seite gleichsam in einem Verhältnis der Nullsummenkonkurrenz zueinander stehen: Was sozialintegrativ funktional ist, ist im Hinblick auf Systemintegration und ökologische Integration dysfunktional, und umgekehrt.

Außer durch Ansprüche vermag die Person in der modernen Gesellschaft ihre Identität, wie auch schon in vormodernen Gesellschaften, weiterhin durch die Bindung an partikulare kulturelle Gemeinschaften zu finden. Das können religiöse, weltanschauliche, ethnische oder durch bestimmte Lebensstile oder Interessen konstituierte Gemeinschaften sein. Es mag sich um kleine Gruppen in der jeweiligen Population, also 
z.B. Sekten, handeln, oder um quantitativ gewichtigere Subkulturen oder auch um regionale oder nationale Mehrheiten, z.B. die Basken im Baskenland oder die Islamisten im Iran. ${ }^{15}$ In jedem Fall zieht der jeweilige Partikularismus Grenzen der Zugehörigkeit, die sowohl sachlich als auch sozial identitätsstiftend wirken. Sachlich wird ein eingeschränkter, damit überschaubarer und in sich geschlossener Sinnzusammenhang konturiert, der einen Rahmen aller als wesentlich erachteter Fragen und Antworten schafft. Sozial wird dies dadurch stabilisiert, dass diesen Sinnzusammenhang bestätigende ingroup-Interaktionen überwiegen, in denen dann überdies alle nicht Dazugehörigen pauschal, und meist einige andere Gemeinschaften gezielt, hinsichtlich ihrer Lebensstile und Einstellungen abgewertet werden. Immer wieder bilden sich so einander bis zur ungerührten oder gar enthusiastischen Gewalttätigkeit feindselig gesinnte Paare solcher Gemeinschaften, wofür die Protestanten und Katholiken in Irland nur ein besonders extremes Beispiel abgeben. Für den deutschen nationalen Kontext hat dies die Forschergruppe um Heitmeyer (1997a; 1997b) besonders prägnant herausgebildet, für die „Weltgesellschaft" stellt Samuel Huntington (1996) hierzu weitreichende Überlegungen an, die insbesondere durch den islamischen Fundamentalismus angeregt worden sind. In Rechnung zu stellen sind dabei auch die Affekte von Modernisierungsverlierern, also gesellschaftlichen Gruppen, deren sozialer Status durch eine fortschreitende Ausdifferenzierung der Teilsysteme gefährdet und abgewertet wird. Was systemintegrativ durchaus bewältigbar ist, muss sozialintegrativ noch lange nicht verkraftet werden.

In dem Maße, wie die jeweilige Gruppen-Identität, auf der die je individuellen Identitäten der Dazugehörigen basieren, sich erst über die Gegnerschaft zu anderen Gemeinschaften profiliert, ist ein gesellschaftliches Konfliktpotential angelegt. Dieses auf einer zur Polarität zugespitzten Andersartigkeit beruhende Konfliktpotential ist für sich genommen prinzipieller und damit weniger kompromissfähig, als es Verteilungskonflikte zwischen Besser- und Schlechtergestellten sind. Kulturkonflikte können also als kollektive Identitätskonflikte einerseits schärfere, unberechenbare Eskalationsdynamiken hervorbringen, womit partikulare Gemeinschaften als sozialintegrativer Mechanismus noch problematischer als der Anspruchsindividualismus sein können. Andererseits

15 Zigmunt Bauman (1991) geht so weit, die Bürger moderner Nationalstaaten oder sogar die westlichen Gesellschaften insgesamt als große kulturelle Gemeinschaften anzusehen, die alle Fremden - von Ausländern bis zu Minderheiten mit fremdartigen Lebensstilen - stigmatisieren, ausgrenzen und disziplinieren, um so eine identitätssichernde Normalität zu erhalten. 
kann selbst eine abgrundtiefe Verachtung zwischen den Gemeinschaften auch mit bloßer wechselseitiger Meidung und Indifferenz einhergehen, wie es oft genug zwischen religiösen Sekten der Fall ist. Die sozialintegrativ problematischsten $(\leftarrow$ p.19) Fälle sind daher diejenigen, in denen Kultur- und Verteilungskonflikte korrelieren und einander gegenseitig begründen und anheizen - wie es ja sowohl beim irischen als auch beim islamischen Beispiel nicht zufällig der Fall ist. Dann werden die partikularen Gemeinschaften zu kollektiven Trägern dessen, was ansonsten der Anspruchsindividualismus artikuliert; und die kulturelle Legitimation schürt Maximalforderungen, an denen jede Aushandlung von graduellen und schrittweisen Annäherungen der Konfliktparteien fast zwangsläufig scheitert. Man muss nicht so weit gehen wie Huntington, der im weltweiten „clash of cultures“ letztlich nur noch die militärische Überlegenheit einer Seite als wirksamen sozialintegrativen Mechanismus ansieht, um seine Zustandsdiagnose eines äußerst prekären sozialintegrativen Problems zu teilen.

Wenn somit partikulare kulturelle Gemeinschaften mit ihrer Konstituierung von Identität durch Differenz gegenüber anderen sozialintegrativ höchst ambivalent einzustufen sind, weil sie zwar das Individuum einbinden, dies aber nur dadurch, dass sie es gegen andere aufbringen, könnte der Schluss gezogen werden, dass es derartige Gemeinschaften am besten gar nicht gäbe und man alles tun sollte, was sie schwächt und zum Absterben bringt. Dem würden allerdings soziologische Gegenwartsdiagnosen wie die von Amitai Etzioni (1993; 1996) oder auch die von Sennett (1998) vehement widersprechen. Für sie ist, wie schon für Ferdinand Tönnies (1887), die Schwächung von Gemeinschaften - von Familien über Nachbarschaften und Kirchengemeinden bis zu Interessengruppen und Nationen - die zentrale Ursache schwindender gesellschaftlicher Sozialintegration, wobei darunter vor allem ein rücksichtsloser Egoismus der Gesellschaftsmitglieder verstanden wird, der sich auch in individueller Devianz, wo immer dies straffrei möglich ist, äußert; und nur eine Stärkung der den einzelnen auf das Gemeinwohl verpflichtenden Gemeinschaften kann die Sozialintegration der modernen Gesellschaft sicher stellen. Der Anspruchsindividualismus gilt also gerade nicht als funktionstüchtiger sozialintegrativer Mechanismus, sondern als Haupt-Störenfried der Sozialintegration.

Vor dem Hintergrund der zuvor referierten Befürchtungen über Kulturkonflikte muss man bei derartigen „kommunitaristischen“ Gegenwartsdiagnosen um so genauer 
nachfragen, wie sie sich das Nebeneinander von partikularen Gemeinschaften vorstellen, die unterschiedliche kulturelle Traditionen und Projekte tragen. Etzioni (1997: 251/252) hält diesbezüglich weder den „melting pot“ noch einen simplen Multikulturalismus für realistisch, sondern gebraucht das Bild des „Mosaiks“, also eines durch bestimmte allen gemeinsame Werte gerahmten Pluralismus der Kulturen. Habermas (1992) würde weniger auf Werte als auf öffentliche Diskurse und rechtsstaatliche Prinzipien als tragende Elemente einer „Zivilgesellschaft“ setzen. Zu fragen ist freilich jeweils: Sind dies Wunschvorstellungen, oder gibt es dafür reale Entsprechungen? Und falls nichts davon funktioniert: Ist dann auf Dauer mit Konfliktherden zwischen den verschiedenen Gemeinschaften zu rechnen?

Was Etzioni unmissverständlich heraus arbeitet, ist das letztlich unauflösbare Spannungsverhältnis zwischen Individualisierung im Sinne einer immer stärkeren Selbstbestimmung des eigenen Lebens und Gemeinschaftsbindung. Für inn kann man nicht beides zugleich maximieren; und die Betonung von Individualisierung während $1 \leftarrow$ p.20) der letzten Jahrzehnte sei, an sozialintegrativen Erfordernissen gemessen, übers Ziel hinaus geschossen. Beck (1986) oder Giddens (1990) sehen dies anders. Für sie trägt die Tatsache, dass immer mehr Gesellschaftsmitglieder aufgrund von Bildungsexpansion, veränderten Arbeitsmarktstrukturen, wohlfahrtsstaatlichen Leistungen, räumlicher Mobilität und Wertewandel ein immer selbstbestimmteres Leben führen, zunächst einmal zur gesellschaftlichen Sozialintegration in dem Sinne bei, dass die Individuen sich stärker mit ihrer „Bastel-Biographie“ (Hitzler 1996) identifizieren und dadurch mit der Komplexität der modernen Gesellschaft zurechtkommen. Zwar lösen sich durch Individualisierung traditionelle Gemeinschaften auf; doch neue Arten der Vergemeinschaftung entstehen, etwa die Event-Gemeinschaften der Techno-Szene oder „sub-politische“ Gemeinschaften des kollektiven Kampfs - beispielsweise auch gegen ökologische Risiken (Beck/Beck-Gernsheim 1994: 35). Dass diese Gemeinschaften sozialintegrativ weniger gut funktionieren als traditionelle, wäre erst noch zu zeigen.

Allerdings wird von „riskanten Freiheiten“ (Beck/Beck-Gernsheim 1994) gesprochen, die Individualisierung der Gesellschaftsmitglieder also neben der schon behandelten "organisierten Unverantwortlichkeit" der Teilsysteme als zweite Komponente der „Risikogesellschaft" verbucht. Individuelle Selbstbestimmung impliziert unweigerlich 
erhöhte biographische Risiken, von der Ehescheidung über die Entwertung von Bildungszertifikaten bis hin zur Dauerarbeitslosigkeit. Diese Risiken treffen den einzelnen um so ungehemmter, je mehr er sich aus traditionellen Gemeinschaftsbindungen gelöst hat und je mehr er selbst die Verantwortung auch für biographische Fehlentscheidungen übernehmen muss (Giddens 1990). Und diese Risiken treffen als biographische Externalitäten auch andere, z.B. die Kinder einer geschiedenen Ehe - siehe auch die von Sennett (1998: 21) wiedergegebenen Befürchtungen eines seiner Protagonisten des „flexiblen Kapitalismus“, daß seine Kinder zu „mall rats“ werden könnten. Mehr oder weniger weitreichendes biographisches Scheitern kann, wenn es massenhaft auftritt, sozialintegrativ problematisch werden. Auszumachen ist Derartiges allerdings, jenseits gelegentlicher aufgeregter Berichterstattungen in den sensationshungrigen Massenmedien, bislang nicht, so dass die sozialintegrativ positiven Effekte der Individualisierung in dieser Bilanz überwiegen.

Damit sind die verschiedenen Argumentationsstränge derjenigen soziologischen Gegenwartsdiagnosen dargestellt, die eingetretene oder mögliche Probleme einer zu geringen Sozialintegration sehen. Dem können nun Sichtweisen gegenübergestellt werden, die Gefahren der Überintegration sehen - wobei beides einander nicht notwendig widersprechen muss, weil Des- und Überintegration auch zugleich vorkommen können. Analytisch heißt Überintegration, dass die Gesellschaft den Charakter einer "greedy institution“ (Coser 1974) und zugleich einer „totalen Institution“ (Goffman 1961) annimmt. Letztere, etwa durch eine Kaserne versinnbildlicht, strebt eine völlige Kontrolle des Handelns derer an, die in ihr agieren; erstere - als Beispiel eine fanatische Sekte - erwartet ein grenzenloses Engagement der in ihr Handelnden für sie. Zusammengenommen heißt dies: Die moderne Gesellschaft tendiert in dem Maße zur Überintegration, wie sie einer Kasernierung fanatischer Sektenanhänger gleicht, also totale Kontrolle mit totalem Engagement kombiniert. $(\leftarrow \mathbf{p . 2 1})$

Betrachtet man zunächst die Folgewirkungen von Überintegration beim je einzelnen Gesellschaftsmitglied, lässt sich feststellen, dass die soziologischen Gegenwartsdiagnosen der letzten zwanzig Jahre ein Thema fortführen, das schon von einigen soziologischen Klassikern, insbesondere Weber (1922: 125-130, 551-579), ausgearbeitet worden ist: die Entfremdung des Individuums in der Organisationsgesellschaft. Die Durchdringung fast aller gesellschaftlichen Teilsysteme durch formale Organisationen 
- eine systemintegrativ höchst wichtige Strukturkomponente (Schimank 1997: 48-53) - führt nach Coleman (1982) zu einer „asymmetrischen Gesellschaft“, in der sozusagen die Organisationen die Macht übernehmen und die Individuen sich dem nur noch ohnmächtig fügen können. George Ritzer (1993) ergänzt diese Diagnose durch den Hinweis darauf, dass diese Vormachtstellung der Organisationen in nahezu allen Lebensbereichen zu einer gewissermaßen selbstreferentiellen Verabsolutierung organisatorischer Gesichtspunkte der Effizienz, Routinisierbarkeit, Erwartungssicherheit und Kontrolle führe. Das Individuum wird demzufolge immer mehr zum bloßen Anhängsel organisatorischer Imperative und Vorgänge. Was Weber ebenso wie die klassische Kritische Theorie noch hauptsächlich für die Mitglieder formaler Organisationen aufgezeigt hatten, wird nunmehr von Coleman und Ritzer auch auf diejenigen bezogen, die von außen in Kontakt mit Organisationen treten: Kunden, Klienten, aber auch durch Nebenwirkungen von Organisationshandeln Betroffene wie z.B. die Anwohner eines die Luft verschmutzenden Chemiewerks.

Auf den ersten Blick ist zwar erkennbar, dass die moderne Gesellschaft als Organisationsgesellschaft ein strukturelles Gefährdungspotential hinsichtlich der individuellen Autonomie der Gesellschaftsmitglieder besitzt. Sozialintegrativ erscheint dies aber zunächst gerade nicht als problematisch, sondern als funktional, wird doch scheinbar umfassende Fügsamkeit erzeugt. Bei genauerem Hinsehen entdeckt man freilich, dass diese Fügsamkeit leicht in Apathie und „Dienst nach Vorschrift“ umschlagen kann, weil das Individuum sich selbst - seine Vorstellungen und Fähigkeiten ebenso wie seine Interessen und Bedürfnisse - nicht mehr gesellschaftlich zur Geltung zu bringen vermag, sondern von der Gesellschaft entfremdet ist. Gerade die Forderung nach totalem Engagement kann, wenn mit totaler Kontrolle gepaart, stillschweigendes Disengagement hervorbringen; und die totale Kontrolle sorgt nur dafür, dass um so eifriger und findiger nach Möglichkeiten klammheimlicher Subversion gesucht wird. Im Extremfall stellt sich eine derart überintegrierte Gesellschaft dann als ein Kartenhaus dar, das schon durch kleine Erschütterungen zum Einsturz gebracht werden kann, weil alle Gesellschaftsmitglieder nur noch simulieren, dass sie Träger gesellschaftlicher Ordnung sind. Die ehemaligen sozialistischen Staaten Osteuropas entsprachen in ihrer Verfallsphase ziemlich genau diesem Bild; und das Ende ließ dann bekanntlich auch nicht lange auf sich warten. 
Überintegration hat weiterhin die höchst dysfunktionale Folgewirkung, dass in Interaktionen zwanghafter Konsens hergestellt wird und so innovative Problemlösungen unterdrückt werden. Alain Touraine (1992) spricht dies mit seiner Unterscheidung von "agent" und „actor" an. Der „Agent" ist das bloße vorprogrammierte Exekutivorgan sozialer Vorgaben, insbesondere in Gestalt organisatorischer Regeln. Der „Akteur" hingegen ist das schöpferische Individuum, dessen Streben nach individueller Selbstbestimmung eben immer auch die gesellschaftlich höchst wichtige positive $\mathrm{Ne}-(\leftarrow \mathbf{p . 2 2})$ benwirkung der Produktion von Innovationen aller Art zeitigt. Auch dies fehlte in den ehemals sozialistischen Staaten und trug zu deren Niedergang bei. Verallgemeinert: Wo Widerspruch verpönt und organisatorisch nicht vorgesehen ist, kann nichts Neues entstehen. Die moderne Gesellschaft muss daher, weil sie Organisationsgesellschaft ist und auch aus vielerlei Gründen bleiben muss, beständig darauf achten, dass sie nicht deshalb sozialintegrativ über die Stränge schlägt und sozial unzuverlässige und innovationsscheue Individuen hervorbringt.

Zusammenfassend lässt sich damit zur Sozialintegration festhalten: Sie wird in den soziologischen Gegenwartsdiagnosen der letzten zwanzig Jahre so intensiv wie eh und je thematisiert, und etliche der verschiedenen Themenstränge kontinuieren auch Einschätzungen, die sich bereits viel früher herausgebildet haben. Deutlich wird weiterhin, dass einige sozialintegrativ positive Mechanismen sich zumindest längerfristig höchst negativ auf die Systemintegration und die ökologische Integration der modernen Gesellschaft auswirken könnten. In diesen Wechselbeziehungen zwischen den drei Integrationsdimensionen liegt vielleicht der gefährlichste Sprengstoff für die zukünftige Integration der modernen Gesellschaft.

III

Die Zusammenschau der soziologischen Gegenwartsdiagnosen ergibt ein recht facettenreiches Bild der Integrationsprobleme der modernen Gesellschaft. Die gesellschaftliche Systemintegration stellt sich als insgesamt gewährleistet dar. Doch zum einen 
zahlt die Gesellschaft den Preis dafür teilweise in Form sozialintegrativer Risiken und Probleme. Zum anderen kann umgekehrt die Art, wie die moderne Gesellschaft ihre Sozialintegration sichert, Folgeprobleme für die Systemintegration und auch für die ökologische Integration nach sich ziehen. Letztere ist als dritte Dimension gesellschaftlicher Integration neu ins Blickfeld gerückt, wodurch die auch zuvor schon nicht einfache Integrationsfrage nur noch komplizierter geworden ist.

Überwiegend ergänzen die Beiträge der verschiedenen soziologischen Gegenwartsdiagnosen zur Integrationsfrage einander. Klar erkennbare und offen ausgetragene Widersprüche zwischen Einschätzungen gibt es kaum. Am deutlichsten ist noch die konträre Bewertung von Gemeinschaft und Individualisierung durch verschiedene Beobachter. Man kann daher, wie hier skizziert, Schritt für Schritt die Steine eines Puzzles zusammenfügen und gewinnt so in der Zusammenschau ein informationsreicheres Bild der Integrationsprobleme der modernen Gesellschaft als aus jeder einzelnen Gegenwartsdiagnose. Die Sekundäranalyse liefert damit zu dieser Thematik vor allem eine Wissenskumulation und -integration und präpariert weniger eine dann einer Entscheidung zuführbare Konkurrenz von Deutungsalternativen heraus.

Offen bleibt dabei freilich, welche relative Bedeutsamkeit im Sinne von kausalem Gewicht den verschiedenen Elementen des Modells zugesprochen wird. Tragen bei- $(\leftarrow$ p.23) spielsweise die partikularen kulturellen Gemeinschaften mehr oder weniger zur Sozialintegration bei als der Anspruchsindividualismus, überwiegt der systemintegrative Beitrag der Organisationsgesellschaft ihre sozialintegrativen Folgeprobleme, oder ist die ökologische Integration stärker gefährdet als die Sozialintegration? Spätestens Fragen dieser Art verweisen darauf, dass alles weitere nur empirisch zu klären ist. Theoretisch lassen sich plausible Hypothesen aufstellen und zu immer komplexeren Modellen zusammenfügen - wie es hier getan worden ist. Mehr als das vermag Theorie nicht zu leisten. Letztlich formuliert sie möglichst spezifische Anfragen an entweder bereits vorhandenes oder erst noch zu erarbeitendes empirisches Wissen. Dementsprechend kann die vorgelegte Sichtung der gegenwartsdiagnostischen Thesen zur gesellschaftlichen Integration zum einen dazu anleiten, schon verfügbares empirisches Wissen sekundäranalytisch zur Prüfung einzelner Thesen heran zu ziehen. Zum anderen können die Thesen dort, wo noch kein empirisches Wissen vorliegt, Fragestellungen für entsprechende Forschungsprojekte anregen. $(\leftarrow$ p.24) 


\section{Literatur}

Axelrod, Robert, 1986: An Evolutionary Approach to Norms. In: American Political Science Review 80, 1095-1111.

Bauman, Zigmunt, 1991: Modernität und Ambivalenz. Frankfurt/M., 1992: Fischer.

Beck, Ulrich, 1986: Die Risikogesellschaft. Frankfurt/M.: Suhrkamp.

Beck, Ulrich/Beck-Gernsheim, Elisabeth, 1994: Individualisierung in modernen Gesellschaften - Perspektiven und Kontroversen einer subjektorientierten Soziologie. In: Ulrich Beck/Elisabeth Beck-Gernsheim (Hrsg.), Riskante Freiheiten. Frankfurt/M.: Suhrkamp, 10-39.

Berger, Johannes, 1994: The Economy and the Environment. In: Neil Smelser/Richard Swedberg (eds.), The Handbook of Economic Sociology. Princeton NJ: Princeton University Press, 766-797.

Berger, Peter et. al., 1973: The Homeless Mind. Harmondsworth: Penguin.

Bourdieu, Pierre, 1998: Gegenfeuer. Wortmeldungen im Dienste des Widerstands gegen die neo-liberale Invasion. Konstanz: Universtäts-Verlag Konstanz.

Bourdieu, Pierre et al., 1993: Das Elend der Welt. Zeugnisse und Diagnosen alltäglichen Leidens an der Gesellschaft. Konstanz, 1997: Universitäts-Verlag Konstanz.

Coleman, James S., 1982: Die asymmetrische Gesellschaft. Vom Aufwachsen mit unpersönlichen Systemen. Weinheim, 1986: Beltz.

Coser, Lewis A., 1974: Greedy Institutions. Patterns of Undivided Commitment. New York: Free Press.

Crozier, Michel, 1970: La Societe Bloquee. Paris, 1984: Du Seuil.

Dahrendorf, Ralf, 1979: Lebenschancen. Anläufe zur sozialen und politischen Theorie. Frankfurt/M.: Suhrkamp.

Dahrendorf, Ralf, 1992: Der moderne soziale Konflikt. Essay zur Politik der Freiheit. Stuttgart: Deutsche Verlags-Anstalt.

Etzioni, Amitai, 1993: Die Entdeckung des Gemeinwesens. Ansprüche, Verantwortlichkeiten und das Programm des Kommunitarismus. Frankfurt/M.: Fischer.

Etzioni, Amitai, 1996: Die Verantwortungsgesellschaft. Individualismus und Moral in der heutigen Demokratie. Frankfurt/M., 1997: Campus. 
Giddens, Anthony, 1990: Konsequenzen der Moderne. Frankfurt/M., 1995: Suhrkamp.

Goffman, Erving, 1973: Asyle. Über die soziale Situation psychatrischer Patienten und anderer Insassen. Frankfurt/M.: Suhrkamp.

Gross, Peter, 1994: Die Multioptionsgesellschaft. Frankfurt/M.: Suhrkamp.

Habermas, Jürgen, 1973: Legitimationsprozesse im Spätkapitalismus. Frankfurt/M.: Suhrkamp.

Habermas, Jürgen, 1992: Faktizität und Geltung. Frankfurt/M.: Suhrkamp.

Heimann, Edurad, 1929: Soziale Theorie des Kapitalismus. Theorie der Sozialpolitik. Frankfurt/M. 1980: Suhrkamp.

Heitmeyer, Wilhelm (Hrsg.), 1997a: Was treibt die Gesellschaft auseinander? Frankfurt/M.: Suhrkamp.

Heitmeyer, Wilhelm (Hrsg.), 1997b: Was hält die Gesellschaft zusammen? Frankfurt/M.: Suhrkamp.

Hitzler, Ronald, 1996: Die Bastel-Existenz. In: Psychologie heute 7/96, 3035.

Huntington, Samuel, 1996: Der Kampf der Kulturen. Die Neugestaltung der Weltpolitik im 21. Jahrhundert.München/Wien: Eurpaverlag.

Kaufmann, Franz-Xaver, 1991 : Diskurse über Staatsaufgaben. MxPlanck-Institut für Gesellschaftsforschung, Köln: Discussion Paper 91/5.

Latour, Bruno, 1991: Wir sind nie modern gewesen. Versuch einer symmetrischen Anthropologie. Berlin, 1995: Akademie-Verlag.

Lockwood, David, 1964: Soziale Integration und Systemintegration. In: Wolfgang Zapf (Hrsg.), Theorien des sozialen Wandels. Köln/Berlin, 1969: Kiepenheuer \& Witsch, 124-137.

Luhmann, Niklas, 1981: Identitätsgebrauch in selbstsubstitutiven Ordnungen besonders Gesellschaften. In: Odo Marquard/Karlheinz Stierle (Hrsg.), Identität. Poetik und Hermeneutik VIII. München: Fink, 198-227.

Luhmann, Niklas, 1983: Anspruchsinflation im Krankheitssystem. Eine Stellungnahme aus gesellschaftstheoretischer Sicht. In: Philipp HerderDorneich und Alexander Schuller (Hrsg.), Die Anspruchsspirale. Stuttgart: Kohlhammer, 28-49.

Luhmann, Niklas, 1984: Soziale Systeme. Grundriß einer allgemeinen Theorie. Frankfurt/M.: Suhrkamp. 
Luhmann, Niklas, 1986: Ökologische Kommunikation. Kann die moderne Gesellschaft sich auf ökologische Gefährdungen einstellen? Opladen: Westdeutscher Verlag.

Luhmann, Niklas, 1987: Die gesellschaftliche Differenzierung und das Individuum. In: Niklas Luhmann, Soziologische Aufklärung 6. Die Soziologie und der Mensch. Opladen 1995: Westdeutscher Verlag, 125-141.

Luhmann, Niklas, 1997: Die Gesellschaft der Gesellschaft. Frankfurt/M.: Suhrkamp.

Marx, Karl, 1867: Das Kapital, Bd. 1. Frankfurt/M., 1972: Verlag Marxistische Blätter.

Merton, Robert K., 1949: Social Structure and Anmomy. In: Robert K. Merton, Social Theory and Social Structure. New York, 1968: Free Press, 185214.

Mouzelis, Nicos P., 1997: Social and Systems Integration. Lockwood, Habermas, Giddens. In: Sociology 31, 111-119.

Müller, Hans-Peter, 1997: Sinn deuten. Über soziologische Zeitdiagnostik. In: Merkur 577, 352-357.

Münch, Richard, 1991: Dialektik der Kommunikationsgesellschaft. Frankfurt/M.: Suhrkamp.

Münch, Richard, 1995: Dynamik der Kommunikationsgesellschaft. Frankfurt/M.: Suhrkamp.

Offe, Claus, 1975: Berufsbildungsreform. Eine Fallstudie über Reformpolitik. Frankfurt/M.: Suhrkamp.

Parsons, Talcott/Gerald M. Platt, 1973: The American University. Cambridge MA: Harvard University Press.

Polanyi, Karl, 1944: The Great Transformation. Frankfurt/M., 1978: Suhrkamp.

Reese-Schäfer, Walter, 1996: Zeitdiagnose als wissenschaftliche Aufgabe. In: Berliner Journal für Soziologie 6, 377-390.

Rescher, Nicholas, 1980: Scepticism. Oxford: Rowman \& Littlefield.

Ritzer, George, 1993: Die MacDonaldisierung der Gesellschaft. Frankfurt/M., 1995: S. Fischer.

Schimank, Uwe, 1985: Funktionale Differenzierung und reflexiver Subjektivismus. In: Soziale Welt 36, 421-434. 
Schimank, Uwe, 1997: Organisationsgesellschaft. In: Hagener Materialien zur Soziologie Heft 2/1997, 35-60.

Schimank, Uwe, 1999: Funktionale Differenzierung und Systemintegration der modernen Gesellschaft. In: Jürgen Friedrichs/Wolfgang Jagodzinski (Hrsg.), Soziale Integration, Sonderheft 39 der Kölner Zeitschrift für Soziologie und Sozialpsychologie, Westdeutscher Verlag: Opladen, 47-65.

Schimank, Uwe/Volkmann, Ute (Hrsg.), 2000: Soziologische Gegenwartsdiagnosen I - Eine Bestandsaufnahme. Leske + Budrich: Opladen.

Schulze, Gerhard, 1992: Die Erlebnis-Gesellschaft. Kultursoziologie der Gegenwart. Frankfurt/M.: Campus.

Sennett, Richard, 1998: Der flexible Mensch. Die Kultur des neuen Kapitalismus. Berlin: Berlin-Verlag.

Tönnies, Ferdinand (1887): Gemeinschaft und Gesellschaft. Darmstadt, 1963: Wissenschaftliche Buchgesellschaft.

Touraine, Alain, 1992: Critique of Modernity. Oxford, 1995: Blackwell.

Weber, Max, 1919: Wissenschaft als Beruf. Berlin, 1967: Duncker \& Humblot.

Weber, Max, 1922: Wirtschaft und Gesellschaft. Tübingen, 1972: Mohr. 


\section{Abstract}

Ein prominentes Thema soziologischer Zeitdiagnosen sind nach wie vor Integrationsprobleme der modernen Gesellschaft. Wichtige Gegenwartsdiagnosen der letzten zwanzig Jahre aus Deutschland, Frankreich, Großbritannien und den Vereinigten Staaten werden daraufhin betrachtet, was sie zu den drei Dimensionen gesellschaftlicher Integration - Systemintegration, Sozialintegration und ökologische Integration besagen.

Problems of societal integration still are prominent topics of sociological diagnoses of our time. Important studies of the last two decades from Germany, France, the United Kingdom, and the United States are analyzed with respect to the question, what their messages are concerning the three dimensions of societal integration: social, systems, and ecological integration.

\section{Keywords}

Integration - Sozialintegration - Systemintegration - ökologische Integration - moderne Gesellschaft

\footnotetext{
Autor

Uwe Schimank, Professor für Soziologie an der FernUniversität Hagen. Arbeitsgebiete: soziologische Akteur- und Systemtheorien, Theorien gesellschaftlicher Differenzierung, Wissenschafts- und Sportsoziologie. Neuere Buchveröffentlichungen: Soziologische Gegenwartsdiagnosen I: Eine Bestandsaufnahme (2000, hrsg. mit Ute Volkmann); Handeln und Strukturen (2000); Gesellschaftliche Differenzierung (1999, mit Ute Volkmann).
} 